
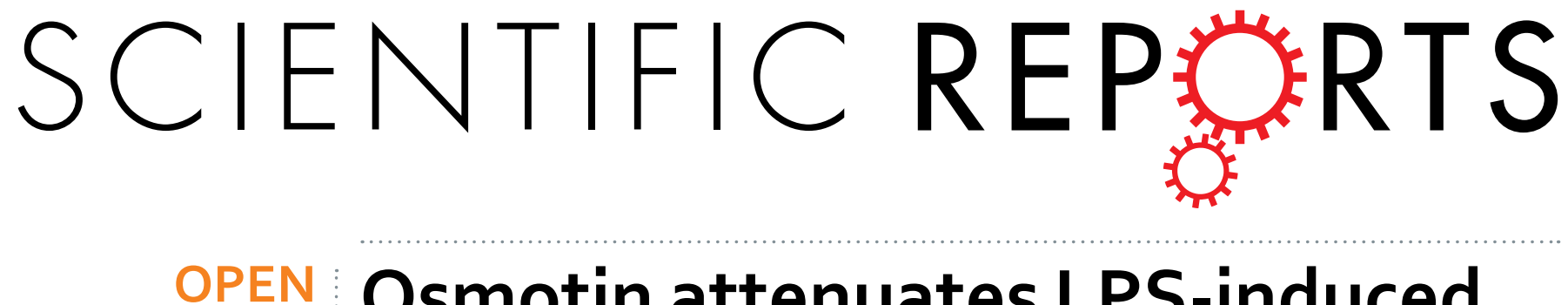

\title{
Osmotin attenuates LPS-induced neuroinflammation and memory impairments via the TLR4/NFKB \\ signaling pathway
}

Received: 08 January 2016

Accepted: 30 March 2016

Published: 20 April 2016

\author{
Haroon Badshah, Tahir Ali \& Myeong Ok Kim
}

Toll-like receptor 4 (TLR4) signaling in the brain mediates autoimmune responses and induces neuroinflammation that results in neurodegenerative diseases, such as Alzheimer's disease (AD). The plant hormone osmotin inhibited lipopolysaccharide (LPS)-induced TLR4 downstream signaling, including activation of TLR4, $C D 14, I K K \alpha / \beta$, and NF $\kappa B$, and the release of inflammatory mediators, such as COX-2, TNF- $\alpha$, iNOS, and IL-1 $\beta$. Immunoprecipitation demonstrated colocalization of TLR4 and AdipoR1 receptors in BV2 microglial cells, which suggests that osmotin binds to AdipoR1 and inhibits downstream TLR4 signaling. Furthermore, osmotin treatment reversed LPS-induced behavioral and memory disturbances and attenuated LPS-induced increases in the expression of AD markers, such as $A \beta$, APP, BACE-1, and $p$-Tau. Osmotin improved synaptic functionality via enhancing the activity of pre- and post-synaptic markers, like PSD-95, SNAP-25, and syntaxin-1. Osmotin also prevented LPS-induced apoptotic neurodegeneration via inhibition of PARP-1 and caspase-3. Overall, our studies demonstrated that osmotin prevented neuroinflammation-associated memory impairment and neurodegeneration and suggest AdipoR1 as a therapeutic target for the treatment of neuroinflammation and neurological disorders, such as AD.

Neuroinflammation is associated with several neurodegenerative disorders, such as Alzheimer's disease (AD), Parkinson's disease, and Huntington's disease. Lipopolysaccharide (LPS) is an endotoxin from the outer membrane of Gram-negative bacteria that activates the immune system. Systemic administration of LPS evokes inflammatory processes in the body, which cause detrimental effects on the brain and other vital organs. In vitro and in vivo studies demonstrated that LPS induced inflammation via the up-regulation of different proinflammatory mediators, such as nitric oxide species (NOS), prostaglandin E2 (PGE2), and cyclooxygenase (COX)-2, and proinflammatory cytokines, including interleukin-1 (IL-1), IL-6, and tumor necrosis factor- $\alpha(\text { TNF- } \alpha)^{1-3}$.

Toll-like receptors (TLRs) play important roles in the sensing of body pathogens and macrophages and the initiation of immune responses. LPS is primarily recognized by the CD14/TLR-4 receptor complex, which is expressed on microglia and astrocytes in the $\mathrm{CNS}^{1,4,5}$. The downstream signal transduction of this receptor complex activates the TLR4/nuclear factor (NF) $\kappa$ B pathway, which leads to the generation of inflammatory mediators and the production of neuroinflammation and neurodegeneration ${ }^{6,7}$. Neuroinflammation induced by a single intraperitoneal (i.p.) injection of LPS persists for 10 months in mouse brain and produces neurodegenerative effects $^{8}$. The neuroinflammatory cytokines and chemokines that are released as result of LPS administration induce amyloidogenesis via an increase in $\beta$-secretase (BACE) activity ${ }^{9}$. Other studies demonstrated a significant increase in the concentration of neuroinflammatory markers, such as proinflammatory cytokines, chemokines, and prostaglandins, in AD brain ${ }^{10,11}$. Earlier studies described an excessive production of IL-1, IL-6, TNF- $\alpha$, macrophage-derived inflammatory factor (MIP)- $\alpha$, and superoxide free radicals in AD glial cells ${ }^{12,13}$. Sambamurti et al. ${ }^{14}$ demonstrated that $\mathrm{NF} \kappa \mathrm{B}$ regulates amyloid beta $(\mathrm{A} \beta)$ formation, and the promoter region of BACE- 1 contains an NFKB binding site ${ }^{14}$. Several studies confirmed that LPS administration impaired spatial learning performance, increased $\mathrm{A} \beta$ production, and disrupted synaptic function ${ }^{15,16}$. Akiyama et al. ${ }^{12}$ demonstrated that transgenic animals overexpressing IL- 6 and TNF- $\alpha$ exhibited cognitive impairment ${ }^{12}$.

Division of Applied Life Science (BK 21), College of Natural Sciences (RINS), Gyeongsang National University, Jinju, 660-701, Republic of Korea. Correspondence and requests for materials should be addressed to M.O.K. (email: mokim@gsnu.ac.kr) 
Several studies demonstrated that different anti-inflammatory agents attenuated the neuroinflammatory effects of LPS ${ }^{16,17}$. However, fewer reports elucidated the exact mechanism of the inhibition of LPS-induced neurotoxicity. Garate et al. ${ }^{6}$ recently demonstrated that an inhibitor of TLR4, TAK242, antagonized the neuroinflammatory effects of LPS ${ }^{6}$. Adiponectin administration to cardiomyocytes interferes with TLR4 signaling and protects against LPS-induced cardiac inflammation ${ }^{18}$. Osmotin is a protein extracted from the tobacco plant that is a structural and functional homolog of the mammalian hormone adiponectin ${ }^{19,20}$. Therefore, we hypothesized that osmotin would interact with the TLR4/CD14 receptor complex and inhibit the downstream inflammatory processes. This study demonstrated that systemic LPS administration induced neuroinflammation and an $\mathrm{AD}$-like pathology in the hippocampus of adult mice. We previously demonstrated the neuroprotective effect of osmotin against ethanol- and amyloid-beta-induced neurotoxicity ${ }^{21,22}$. The present study evaluated the neuroprotective effect of osmotin against LPS-induced neuroinflammation and the possible mechanism of osmotin in the attenuation of the neurotoxic effects of LPS.

\section{Results}

Osmotin prevents LPS-induced neuroinflammatory processes via inhibition of the TLR4-NFKB pathway. The structure-activity relationship of LPS clearly elucidated the binding of LPS with TLR4 receptors, and the crystal structure of the TLR4-MD-2-LPS complex demonstrated the involvement of TLR4 receptor signaling in the progression of LPS-induced inflammatory activities ${ }^{23}$. CD14 receptors also amplify LPS responses, and TLR4/CD14 complexes serve as LPS signaling receptors ${ }^{24}$. Western blot analysis were performed to evaluate the effect of osmotin on LPS-induced activation of TLR4 and CD14 receptors. Our results demonstrated that LPS injection significantly increased TLR4 and CD14 expression compared to the control group in the hippocampus of adult mice and that osmotin treatment significantly reduced the LPS-induced elevated TLR4 and CD14 expression (Fig. 1A).

Several studies demonstrated that LPS-TLR4 signaling mediated the phosphorylation and nuclear translocation of the transcription factor $N F \kappa B^{25}$. IKK $\beta$ phosphorylation is primarily involved in the NFאB activity that is triggered by various proinflammatory stimuli ${ }^{26}$. Western blot and immunofluorescence analyses were performed to explore the anti-inflammatory effect of osmotin on LPS-induced NFKB activation. Western blot analysis revealed that systemic LPS administration significantly increased IKK $\alpha / \beta$ phosphorylation and the nuclear translocation of NFKB-p65 compared to the vehicle-treated group. Osmotin and LPS administration significantly decreased hippocampal levels of $\mathrm{p}-\mathrm{IKK} \alpha / \beta$ and NFKB compared to the LPS-treated group (Fig. 1A). We also demonstrated that osmotin inhibited LPS-induced activation of TLR4 and NFKB in the cortex of adult mice (Fig. S1). NFKB localization after LPS and osmotin treatment was also observed using confocal microscopy. An apparent increase in the CA1, CA3 and DG regions of the hippocampus was observed in LPS-treated mice compared to the control group. Osmotin treatment significantly attenuated LPS-induced NFKB nuclear translocation in the hippocampus compared to the LPS-treated group (Fig. 1B).

Anti-inflammatory mechanism of osmotin against LPS-induced neuroinflammation in BV2 microglial cells. Microglial cells are the primary LPS-responsive cells in the CNS. BV2 microglial cells were used for apoptotic, Western blot, immunoprecipitation, and immunofluorescence assays to elucidate the functional neuroprotective and anti-inflammatory roles of osmotin against LPS-induced neuroinflammation. An Apo-Tox Glo triplex assay kit was used to investigate microglial cell viability, toxicity, and caspase 3/7 activity in control, LPS-treated, osmotin-treated, and LPS and TAK242-treated groups. TAK242 is a well-known inhibitor of the TLR4 receptor, and it inhibits the downstream neuroinflammation following TLR4 activation ${ }^{13}$. Our results demonstrated that osmotin possessed potent neuroprotective activity following LPS treatment via the maintenance of cell viability, reduction of cytotoxicity, and inhibition of caspase 3/7 activation, similarly to LPS and TAK242 treatment. We also demonstrated that $0.4 \mu \mathrm{M}$ osmotin was the optimum dose for adequate neuroprotective activity of the three doses used (i.e., $0.2 \mu \mathrm{M}, 0.4 \mu \mathrm{M}$, and $0.8 \mu \mathrm{M}$ ) (Fig. $2 \mathrm{~A}$ ).

Western blot analysis and immunoprecipitation were performed in microglial cells to investigate the anti-inflammatory mechanisms of osmotin. Osmotin significantly attenuated LPS-induced increases in TLR4, p-NFK B, and TNF- $\alpha$ in microglial cells (Fig. 2B), which supported the anti-inflammatory activity of osmotin. Our immunoprecipitation results demonstrated an interaction of AdipoR1 with TLR4 and its co-receptor CD14 in control, LPS-treated, and LPS plus osmotin-treated microglial cells. Our results demonstrated that LPS treatment enhanced the protein levels of AdipoR1 and the TLR/CD14 complex. We demonstrated that osmotin binding to AdipoR1 inhibited the downstream signaling of TLR4 by dissociating the ligand-receptor complex from the major TLR4/CD14 receptor complex (Fig. 2C). The inhibition of TLR4 downstream signaling was further evaluated using small interfering RNAs (siRNAs) of AdipoR1 treatment to microglial cells, and our results demonstrated that AdipoR1siRNA treatment significantly inhibited the expression of LPS-induced inflammatory markers, such as TLR4 and p-NFKB (Fig. 2D). We also demonstrated an enhanced expression of AdipoR1 in BV2 microglial cells following osmotin and LPS treatment (Fig. S2).

We performed double-labeled immunofluorescence to assess localization and distributional changes of TLR4 and AdipoR1 in microglial cells. Our results revealed the colocalization of TLR4 and AdipoR1 receptors as a clear overlap of fluorescent signals of these receptors in different treatment groups. Immunofluorescence results also demonstrated an increased expression of TLR4 and decreased expression of AdipoR1 in LPS-treated groups compared to the control group. Notably, osmotin reversed the LPS-induced expression trends of TLR4 and AdipoR1 and decreased TLR4 expression and increased AdipoR1 expression compared to the LPS-treated group in microglial cells (Fig. 2E). Overall, these results support the involvement of AdipoR1 in the downstream signal processing of the TLR4/CD14 receptor complex. 


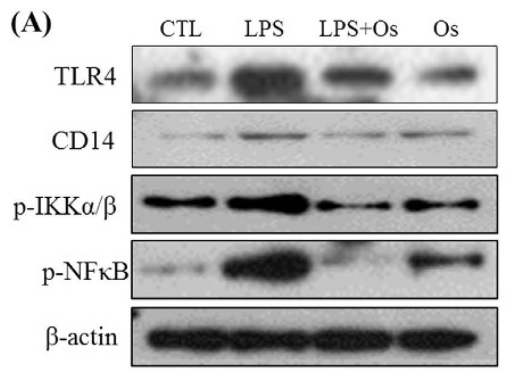

(B)
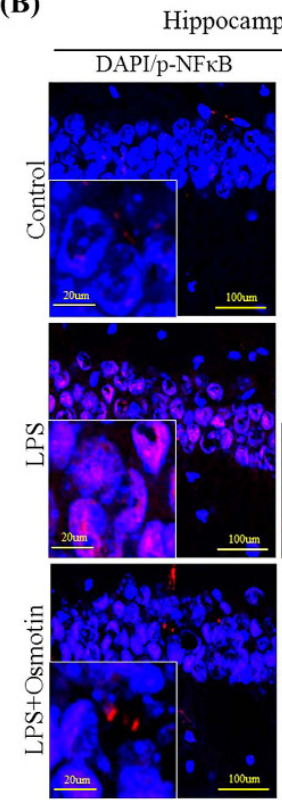
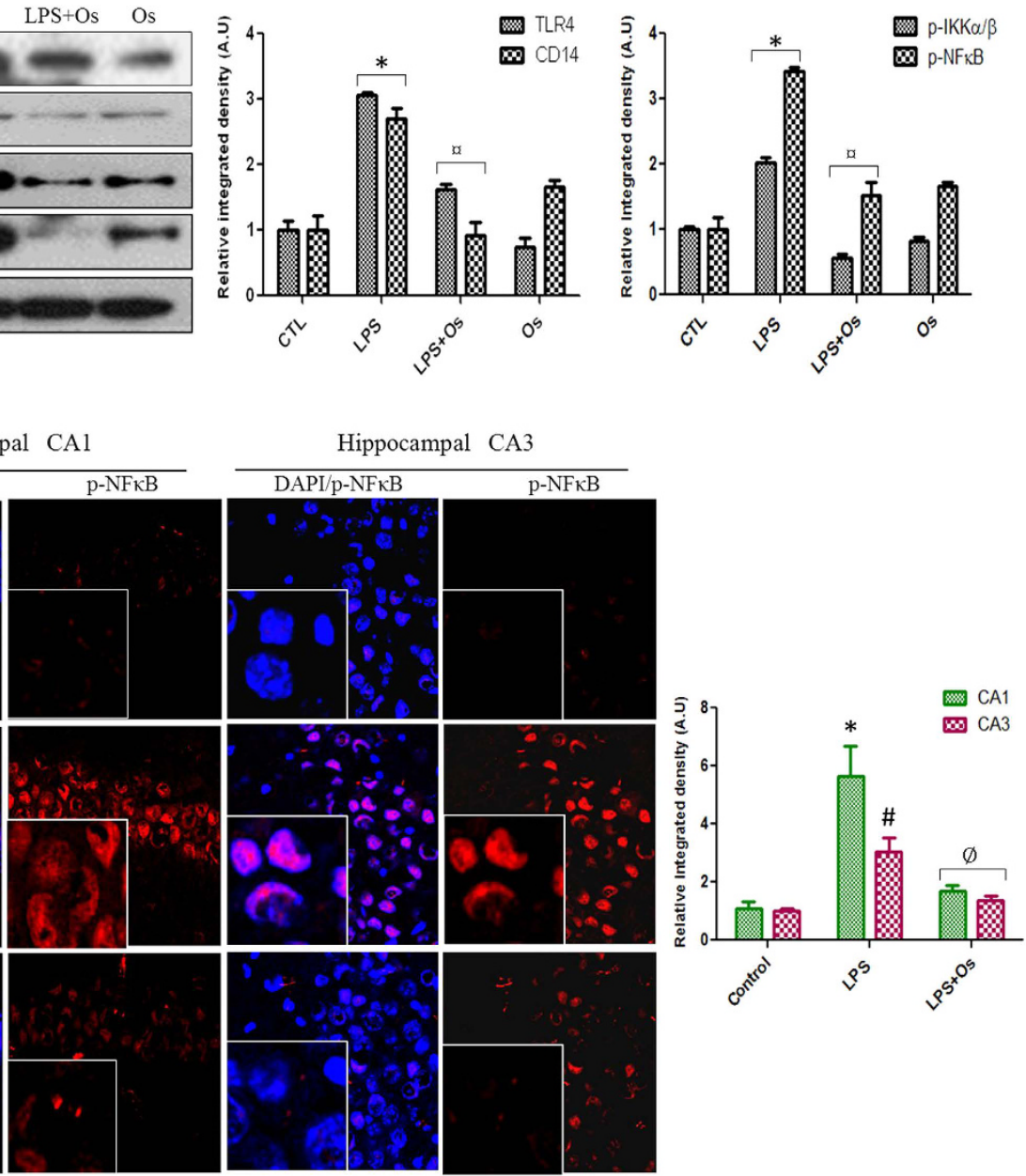

Figure 1. Osmotin prevents LPS-induced neuroinflammatory processes via inhibition of the TLR4NFKB pathway (A) Shown are representative Western blots probed with TLR4, CD14, p-IKK $\alpha / \beta$, and $\mathrm{p}-\mathrm{NF} \kappa \mathrm{B}$ antibodies from the hippocampus of adult mice. The density values are expressed in arbitrary units as the mean \pm SEM of the indicated proteins ( $n=5$ animals per group). (B) Shown are representative photomicrographs of immunofluorescence analyses of p-NFkB-positive cells in the experimental groups. Images are representative stains obtained in sections prepared from at least 5 animals per group. All of the

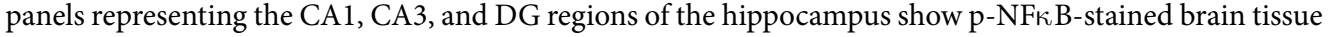
at a magnification of $10 \times$ objective field $($ scale bar $=100 \mu \mathrm{m})$ and magnification of $40 \times$ objective field (scale bar $=20 \mu \mathrm{m}$ ). Symbol representation for the treatment groups and level of significance are described in the data analysis section of the Materials and Methods.

Osmotin inhibits LPS-induced COX-2, iNOS, TNF- $\alpha$, and IL-1 $\beta$ expression. NFkB activation is the primary event in the progression of the proinflammatory signaling pathway. LPS administration induced iNOS activation and the release of proinflammatory mediators, such as TNF- $\alpha$ and IL-1 $\beta$, via the NFKB-MAPK pathway ${ }^{27}$. Font-Nieves et al. ${ }^{3}$ demonstrated that COX-2 activation followed an NFKB-dependent pathway in LPS-treated samples ${ }^{3}$. Hippocampal protein expression levels of COX-2, iNOS, TNF- $\alpha$, and IL-1 $\beta$ were evaluated to examine the effects of LPS and osmotin on the release of inflammatory mediators. Our results demonstrated that LPS treatment for the indicated period induced a higher level of protein expression compared to the vehicle-treated group, and osmotin administration decreased the protein levels of these inflammatory markers in the hippocampus of LPS-injected mice (Fig. 3A). Immunofluorescence detection of TNF- $\alpha$ revealed that osmotin effectively attenuated LPS-induced increases of TNF- $\alpha$ levels in the DG, CA1, and CA3 regions of the hippocampus (Fig. 3B).

Effects of osmotin on LPS-induced activation of astrocytes and microglia in the hippocampus of adult mice. The activation of microglia and astrocytes are the major elements for the progression of neuroinflammation, and activated glial cells are pools for the released inflammatory cytokines ${ }^{28,29}$. LPS treatment activates microglia and astrocytes in vitro or in vivo ${ }^{2,30}$. Therefore, we investigated the inhibitory effect of osmotin on LPS-induced astrocytes and microglial activation. Confocal microscopy revealed that systemic LPS administration significantly increased the number of activated astrocytes (GFAP-reactive cells) and microglia 
(A)
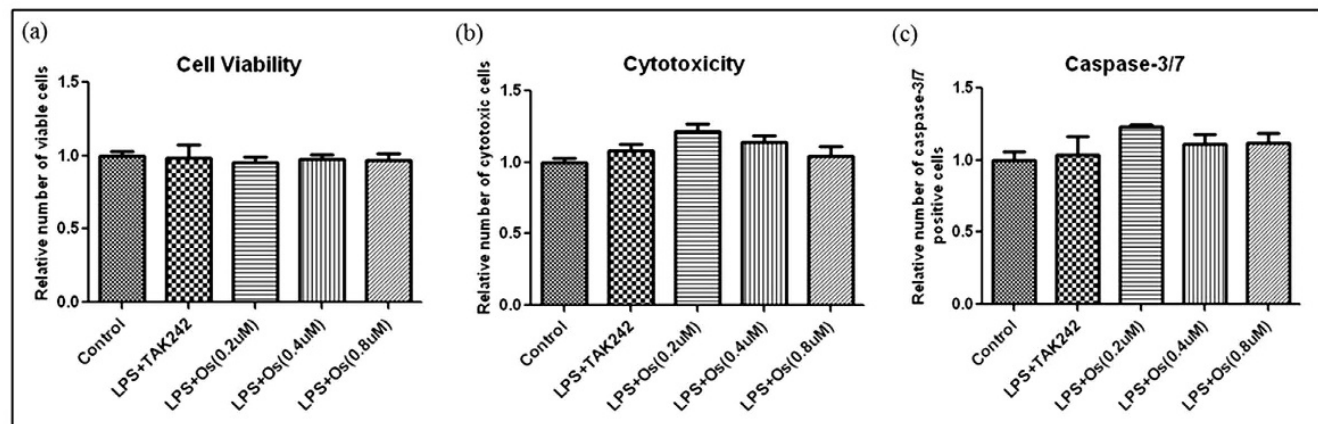

(B)
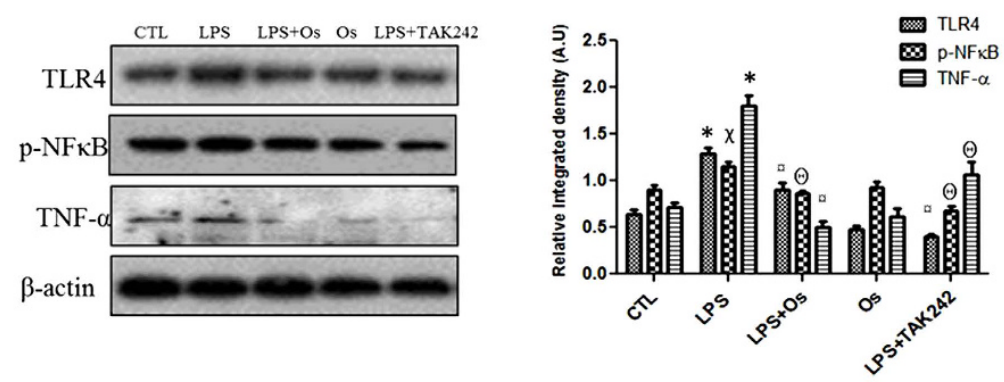

(C)

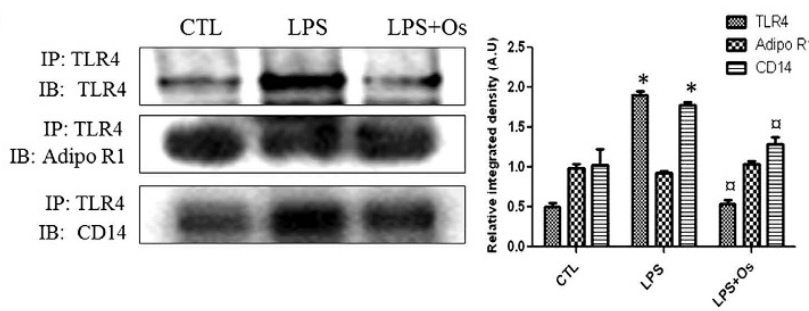

(D)

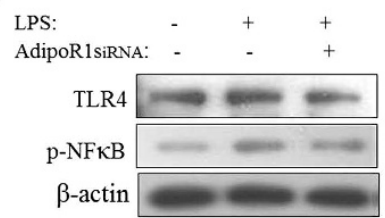

(E)
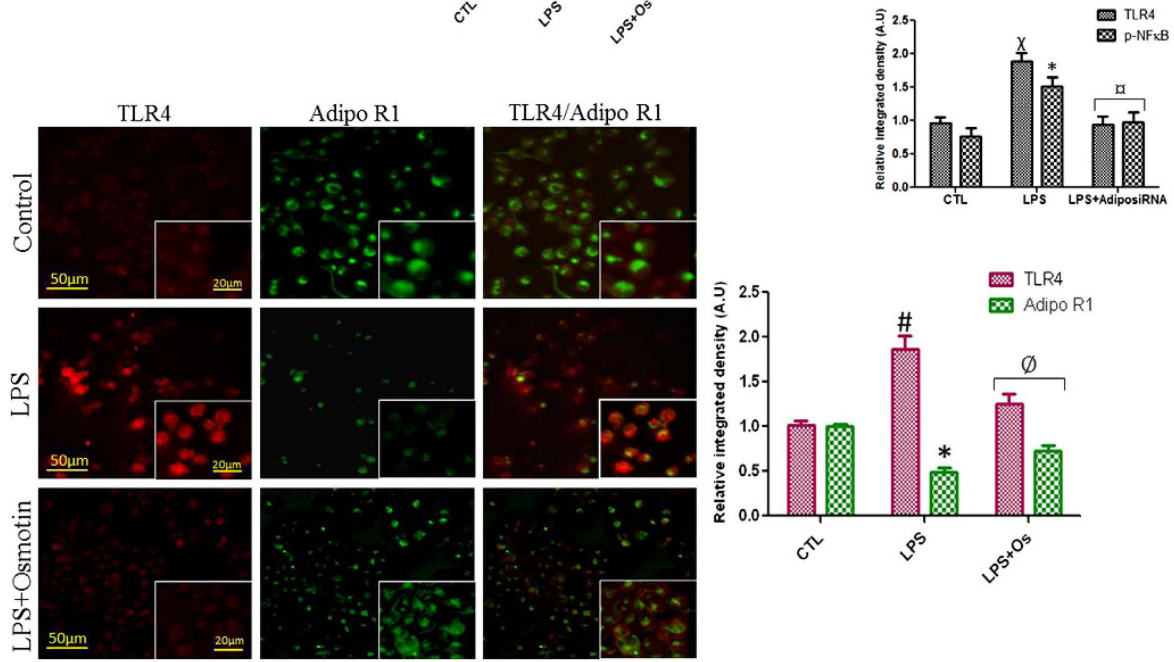

Figure 2. Anti-inflammatory mechanism of osmotin against LPS-induced neuroinflammation in BV2 microglial cells (A) Shown are representative histograms for (a) Cell Viability, (b) Cytotoxicity, and (c) Caspase-3/7 assays performed under experimental conditions as mentioned in the Materials and Methods section. (B) Shown are representative Western blots probed with TLR4, p-NFKB, and TNF- $\alpha$ antibodies in microglial cells. (C) Shown are immunoprecipitation results followed by representative immunoblots as mentioned in the Materials and Methods section. AdipoR1 colocalizes with the TLR4/CD14 signaling complex in microglial cells. (D) Shown are representative Western blots probed with TLR4 and p-NFk B antibodies in microglial cells. AdipoR1 siRNA transfection significantly inhibited LPS-induced inflammation. The density values are expressed in arbitrary units as the mean \pm SEM for the indicated proteins ( $\mathrm{n}=5$ per group).

(E) The immunofluorescence images indicate localization of TLR4 (red panels), AdipoR1 (green panels) and merged TLR4 and AdipoR1 (merge panels). The images are representative of staining obtained in sections prepared from at least 5 animals per group (low magnification $=10 \mathrm{x}$, scale bar: low zoom $=50 \mu \mathrm{m}$ and high zoom $=20 \mu \mathrm{m}$ ). Symbols for treatment groups and levels of significance are mentioned in the data analysis section of the Materials and Methods. 
(A)
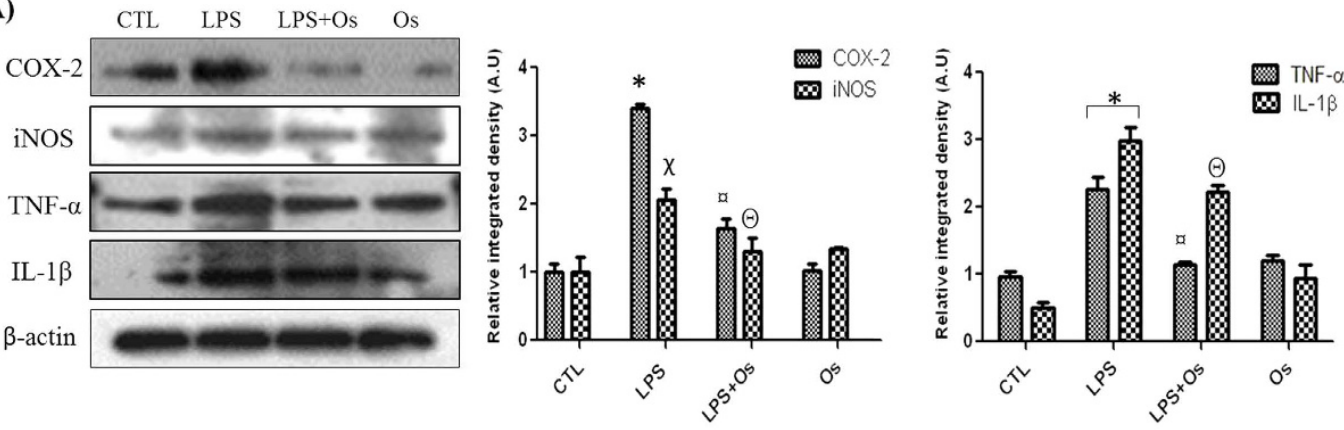

(B)
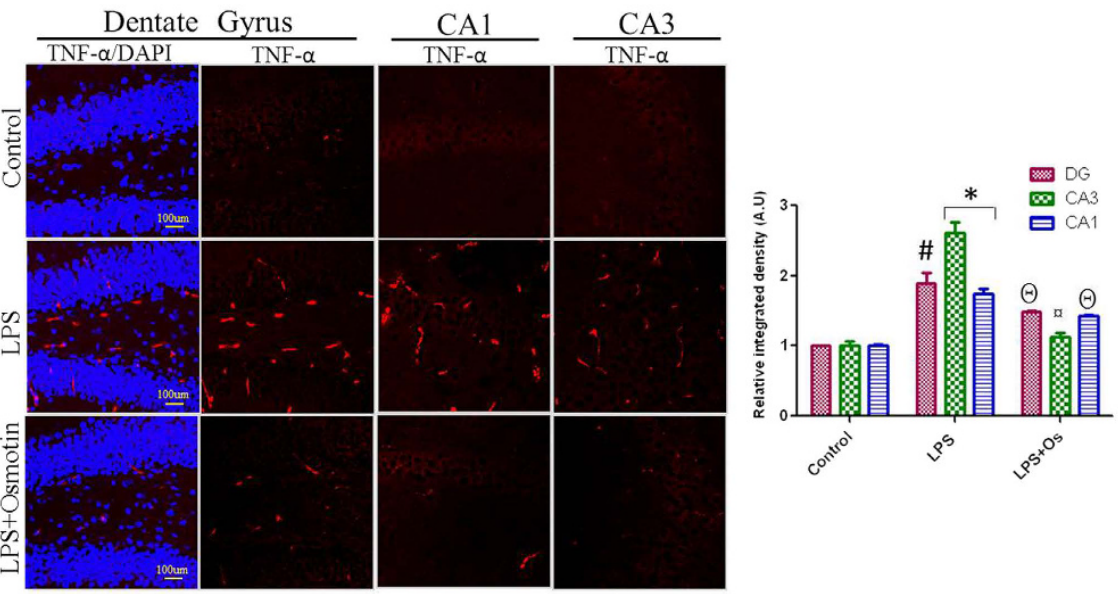

Figure 3. Osmotin inhibits LPS-induced COX-2, iNOS, TNF- $\alpha$ and IL- $1 \beta$ expression (A) Shown are representative western blots probed with antibodies of COX-2, iNOS, TNF- $\alpha$ and IL- $1 \beta$ in the hippocampus of adult mice. The protein bands were quantified using sigma gel software. The density values are expressed in arbitrary units as the mean \pm SEM for the indicated proteins ( $n=5$ animals per group). (B) Showed are representative photomicrographs of immunofluorescence analysis of Tnfa positive cells in the experimental groups. Images are representative of staining obtained in sections prepared from at least 5 animals per group. Panels representing DG, CA1 and CA3 region of hippocampus showed TNF- $\alpha$ stained brain tissue at magnification $10 \times$ objective field, scale bar $=100 \mu \mathrm{m}$. Symbols for treatment groups and level of significance are mentioned in data analysis section of material methods.

(Iba-1-reactive cells) in the cortex and hippocampus of adult mice. Osmotin treatment attenuated the expression of GFAP- and Iba-1-reactive cells compared to the LPS-treated group (Fig. 4).

Osmotin inhibits LPS-induced protein expression of A $\beta, A P P, B A C E-1$, and p-Tau. We examined the effect of LPS and osmotin on the protein expression levels of AD markers, such as A $\beta$, APP, p-Tau, and BACE-1, in the hippocampus of adult mice. Several studies confirmed that LPS treatment for 1 week in adult rats and mice induced AD-like effects, disrupted synaptic function and impaired memory performance ${ }^{4,16}$. Western blots demonstrated that systemic LPS administration significantly increased A $\beta$ and APP levels compared to the control group, and osmotin treatment significantly reversed LPS-induced increases in A $\beta$ and APP levels (Fig. 5A). Our morphological results also demonstrated the anti-AD effect of osmotin. Decreased A $\beta$ levels were observed in the LPS plus osmotin-treated group compared to the LPS-treated group (Fig. 5B). Tau hyperphosphorylation and BACE-1 activation are also critical factors in $\mathrm{AD}$ progression. Therefore, we investigated the expression levels of Tau and BACE-1 in LPS- and osmotin-treated mice. Western blot analysis revealed that systemic LPS administration increased the protein expression of $\mathrm{p}$-Tau and BACE-1 enzyme compared to the control group, and osmotin treatment attenuated LPS-induced Tau hyperphosphorylation and BACE-1 enzyme levels (Fig. 5A). These results were also demonstrated in cortical tissue, which also exhibited a significant reduction in $A \beta$ and p-Tau protein expression following osmotin treatment (Fig. S3).

Effect of osmotin on LPS-induced synaptic dysfunction and memory impairment. Recent studies demonstrated that disruption of synaptic function is a primary feature of $\mathrm{AD}$ and causes cognitive dysfunction and memory impairment with or without the induction of neurodegeneration ${ }^{31}$. Therefore, we investigated the effect of LPS and osmotin on postsynaptic (PSD-95) and presynaptic proteins, such as synaptophysin (SYP), synaptosomal associated protein (SNAP-25), and syntaxin-1. Western blot analyses revealed that systemic LPS administration decreased protein levels of PSD-95, SYP, SNAP-25, and syntaxin-1 in the hippocampus of adult mice compared to the control group. Osmotin treatment reversed the LPS-induced effect on synaptic markers and increased the expression of PSD-95, SYP, SNAP-25, and syntaxin-1 compared to the LPS-treated group (Fig. 6A). 
(A)
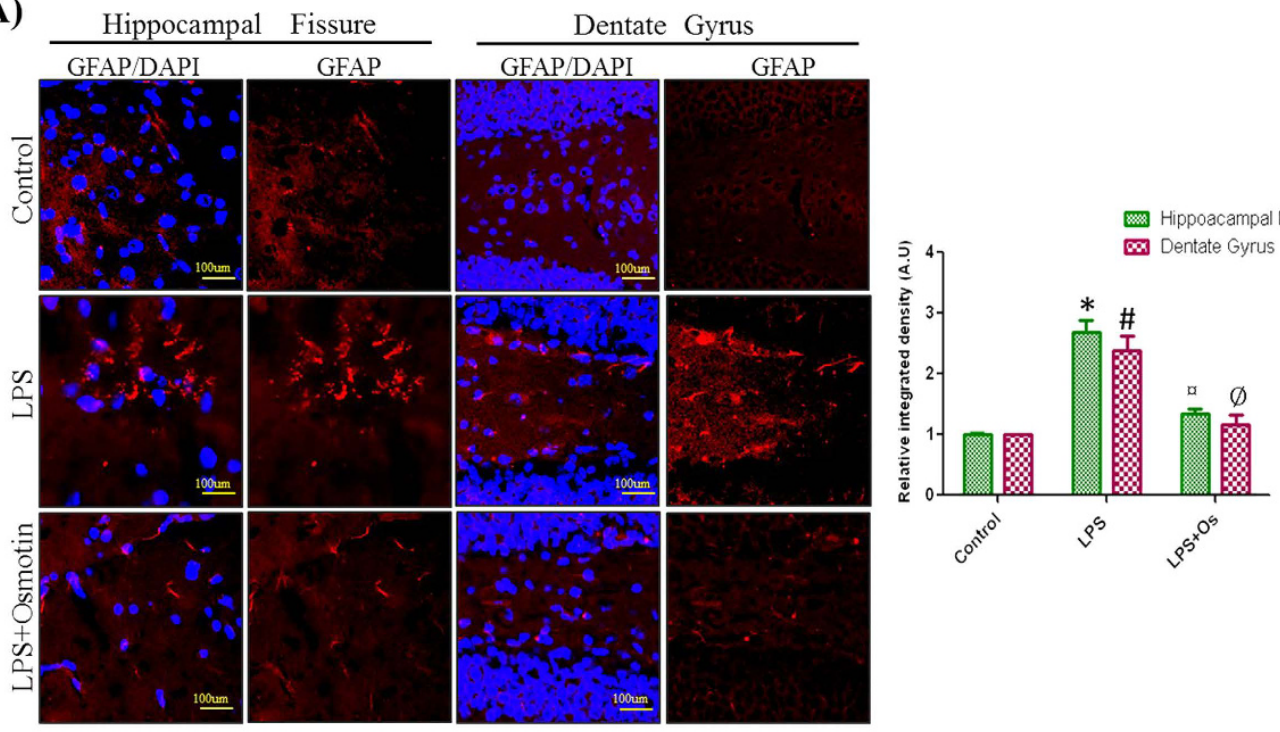

(B)
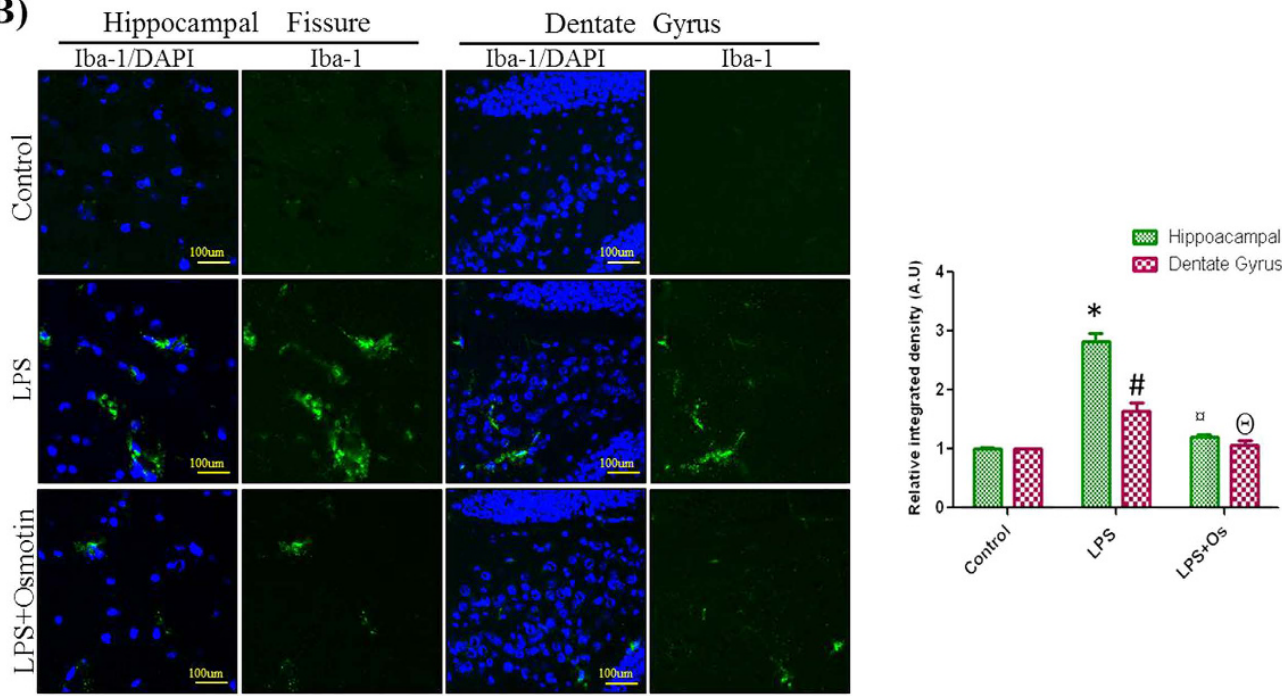

Figure 4. Effect of osmotin on LPS-induced activation of astrocytes and microglia in the hippocampus of adult mice. Shown are representative photomicrographs of immunofluorescence analyses of (A) astrocytes (GFAP-positive cells) and (B) microglia (Iba-1-positive cells) in the experimental groups. Images are representative of staining obtained in sections prepared from at least 5 animals per group. Panels representing the hippocampal fissure and DG region of the hippocampus show GFAP and Iba-1 stained brain tissue, respectively, at a magnification of $10 \mathrm{x}$ objective field, scale bar $=100 \mu \mathrm{m}$. Symbols for the treatment groups and levels of significance are mentioned in the data analysis section of the Materials and Methods.

Immunofluorescence analyses also demonstrated the protective effect of osmotin on LPS-induced synaptic dysfunction. Confocal microscopy revealed a decrease in the fluorescence signals of SNAP-25 and PSD-95 in the hippocampus of the LPS-treated group compared to the control group, and LPS plus osmotin-treated mice exhibited an increased expression of SNAP-25 and PSD-95 compared to the LPS-treated group (Fig. 6B).

Mice were treated with vehicle, LPS, and osmotin, as described in the drug treatment schedule, to evaluate the memory-improving effect of osmotin (Fig. S4). All animal groups were trained to find the hidden platform in a water pool for 4 days, and the Morris water maze test was performed for 6 consecutive days. Our results demonstrated that LPS-treated mice required more time to find the hidden platform and performed fewer platform crossings compared to the control group. The osmotin-treated group required less time to find the hidden platform and performed increased platform crossings compared to the LPS-treated group. The vehicle-treated group exhibited shorter average escape latencies of $46 \mathrm{sec}$ (day first) to $14 \mathrm{sec}$ (day six), and the LPS-treated group exhibited average escape latencies of $49 \mathrm{sec}$ (day first) to $37 \mathrm{sec}$ (day six). These results demonstrate memory impairment in the LPS-treated group. Osmotin treatment significantly ameliorated the LPS-induced cognitive dysfunction, and these mice exhibited shorter average escape latencies of $50 \mathrm{sec}$ (day first) to $23 \mathrm{sec}$ (day six) (Fig. 6C). 
(A)
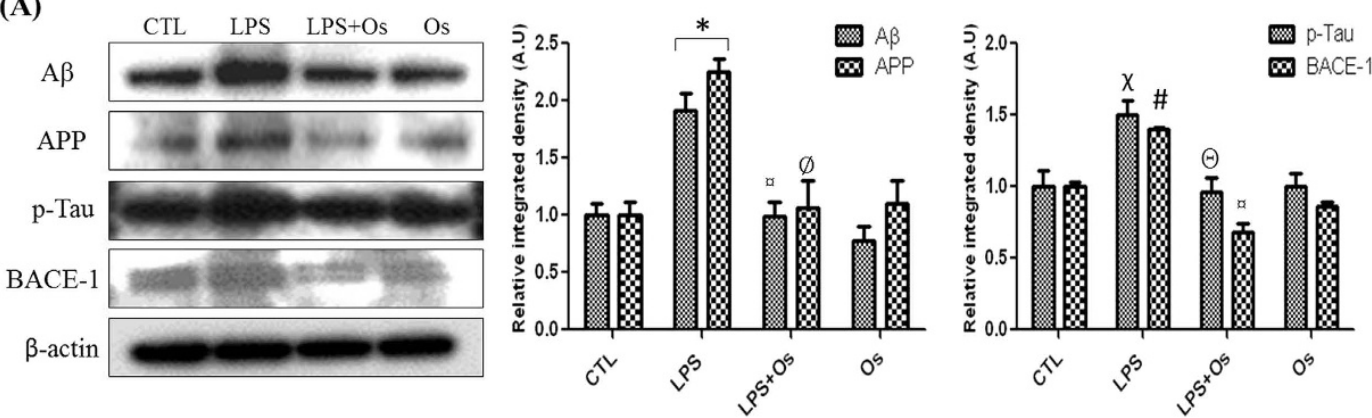

(B)
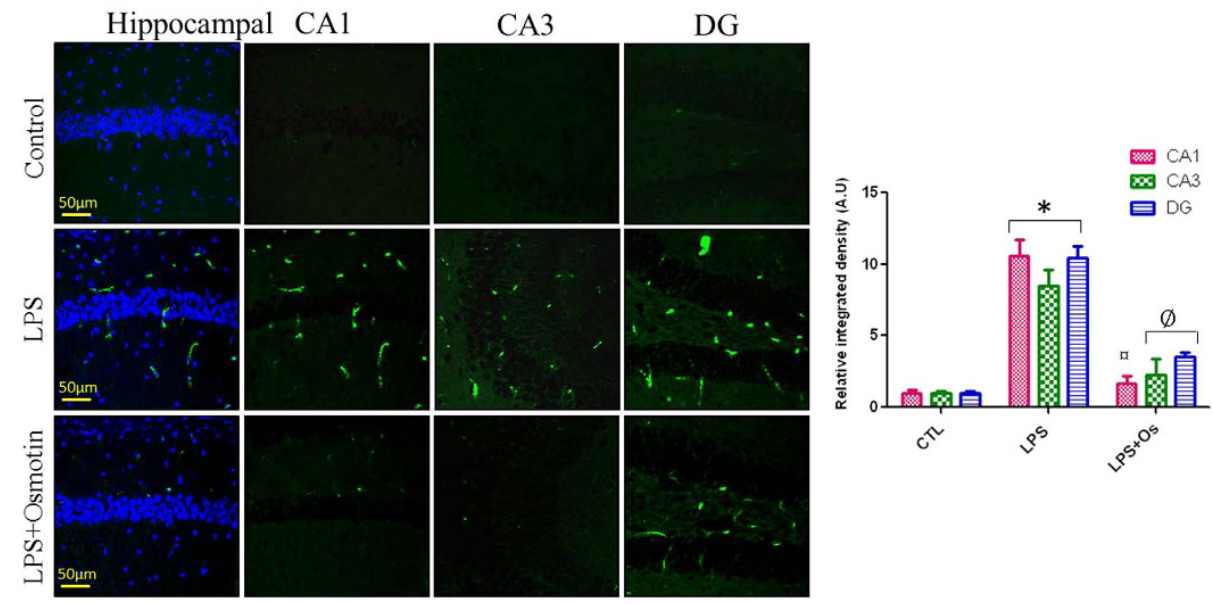

Figure 5. Osmotin inhibits LPS-induced protein expression of A $\beta$, APP, BACE-1, and p-Tau. (A) Shown are representative Western blots probed with $\mathrm{A} \beta$, APP, BACE-1, and $\mathrm{p}$-Tau antibodies in the hippocampus of adult mice. The density values are expressed in arbitrary units as the mean \pm SEM for the indicated proteins ( $n=5$ animals per group). (B) Shown are representative photomicrographs of immunofluorescence analyses of $A \beta$-positive cells in the experimental groups. Images are representative of staining obtained in sections prepared from at least 5 animals per group. All of the panels representing the CA1, CA3, and DG regions of the hippocampus show $\mathrm{A} \beta$-stained brain tissue at a magnification of $10 \mathrm{x}$ objective field, scale bar $=50 \mu \mathrm{m}$. Symbols for treatment groups and levels of significance are mentioned in the data analysis section of the Materials and Methods.

A probe trial was performed $24 \mathrm{hrs}$ after the water maze test in all experimental groups. The hidden platform was removed during this trial, and the average time spent in the target quadrant was measured. LPS-injected mice swam across the entire pool and spent less time in the target quadrant than the control group. Osmotin plus LPS treatment retained the LPS-induced memory impairment because these mice exhibited a significant increase in the average time spent in the target quadrant (Fig. 6C).

Osmotin attenuates LPS-induced apoptotic neurodegeneration in the hippocampus of adult mice. Autocrine secretion of TNF- $\alpha$ and the increased production of NO are the predominant factors of LPS-induced apoptosis ${ }^{32}$. We investigated caspase-3 and PARP-1 protein expression to evaluate the effect of osmotin on LPS-induced apoptotic neurodegeneration. Caspase- 3 is the primary executioner of apoptosis ${ }^{33}$, and PARP-1 overexpression induces DNA damage that is involved in the apoptotic pathway ${ }^{34}$. Our results demonstrated that osmotin administration significantly reduced LPS-induced elevated levels of caspase-3 and PARP-1, which supports its potential neuroprotective capability (Fig. 7A).

The neuroprotective effect of osmotin was investigated morphologically using FJB and Nissl staining in the cortex and hippocampus of adult mice. FJB is a well-known marker for degenerating neurons, and it was used to observe the level of apoptotic neurodegeneration in LPS- and osmotin-treated animals. Our results revealed that LPS treatment significantly elevated the number of degenerated neuronal cells, and osmotin treatment significantly reduced LPS-induced neurodegeneration, which was indicated by the decreased number of FJB-positive cells compared to the LPS-treated group (Fig. 7B). Nissl staining also revealed increased neuronal viability in the osmotin-treated animals compared to LPS-treated animals. Histology of the cortex and hippocampus revealed that LPS treatment increased the level of damaged, fragmented or dead neurons compared to the control group. Osmotin plus LPS administration significantly reduced the number of degenerated neurons in the cortex and hippocampus of adult mice (Fig. 7C). 

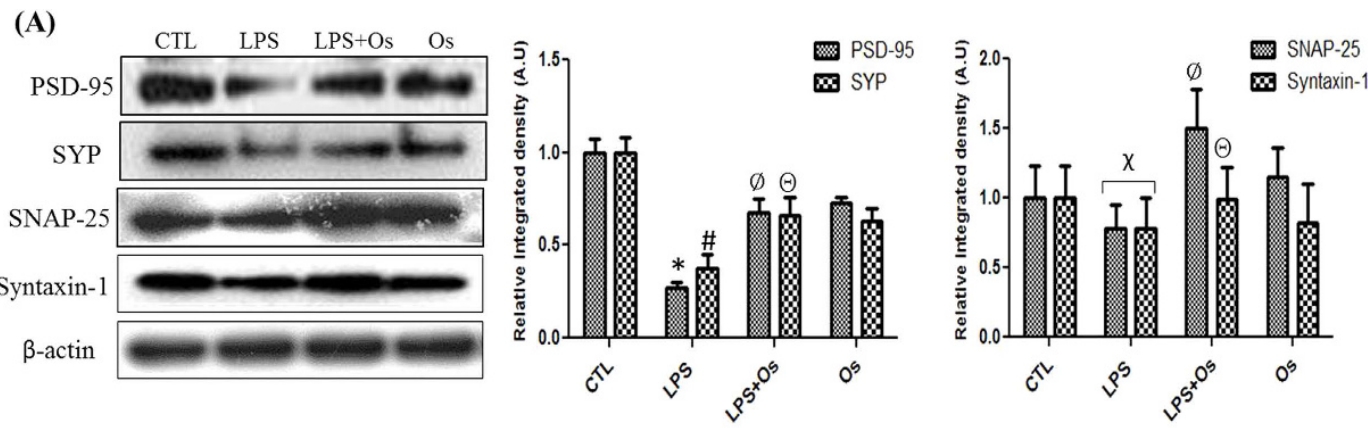

(B)
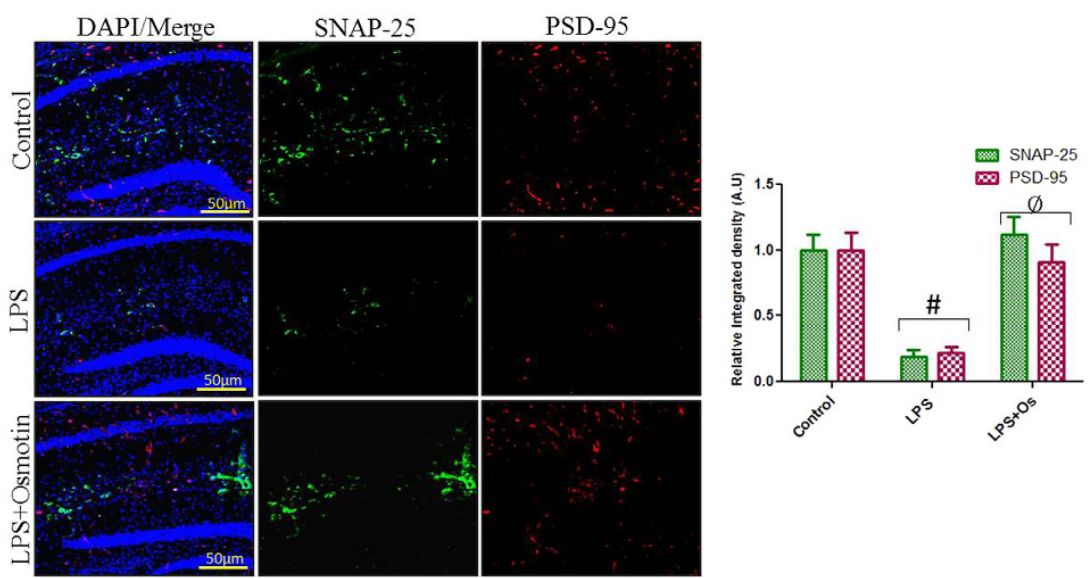

(C)
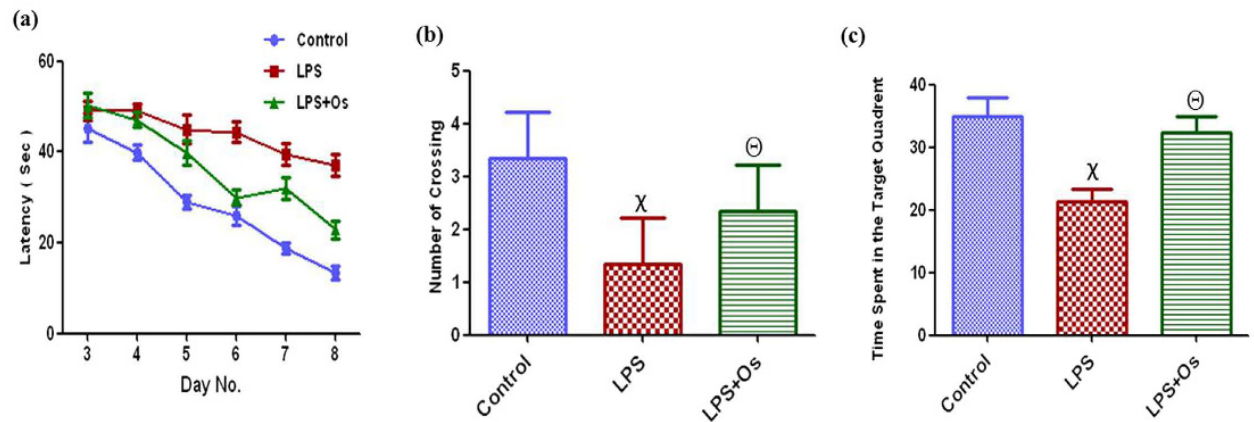

Figure 6. Effect of osmotin on LPS-induced synaptic dysfunction and memory impairment.

(A) Shown are representative Western blots probed with PSD-95, SNAP-25, SYP, and syntaxin-1 antibodies in the hippocampus of adult mice. The density values are expressed in arbitrary units as the mean \pm SEM for the indicated proteins ( $\mathrm{n}=5$ animals per group). (B) Shown are representative photomicrographs of immunofluorescence analysis of PSD-95- and SNAP-25-positive cells in the experimental groups. Images are representative of staining obtained in sections prepared from at least 5 animals per group. All of the panels representing the hippocampus show PSD-95- and SNAP-25-stained brain tissue at a magnification of 10x objective field, scale bar $=50 \mu \mathrm{m}$. Symbols for treatment groups and level of significance are mentioned in the data analysis section of the Materials and Methods. (C) Behavioral studies show osmotin improves memory impairment in LPS-treated mice ( $n=5$ per group). (a) Average escape latency time for experimental mice to reach the hidden platform from day 3 to day 8 . (b) The number of crossings at the hidden platform during the probe test of the Morris water maze experiment. (c) Time spent in the platform quadrant, where the hidden platform was placed during the trial session. Symbols for treatment groups and levels of significance are mentioned in the data analysis section of the Materials and Methods.

\section{Discussion}

The pathogenesis of LPS-induced neuroinflammation and its associated diseases suggest that our therapeutic drug should effectively inhibit the inflammatory processes involving neuronal and microglial cells. Activated microglia acquire macrophage-like functions during neuroinflammation that include phagocytosis and cytokine production $^{35}$. We previously reported that osmotin may ameliorate ethanol- and $\mathrm{A} \beta$-induced neurodegeneration and synaptic dysfunction in rodent brain ${ }^{21,22}$. The present study evaluated the neuroprotective potential of osmotin against neuroinflammation and its associated amyloidogenesis and apoptotic neurodegeneration in an LPS 
(A)
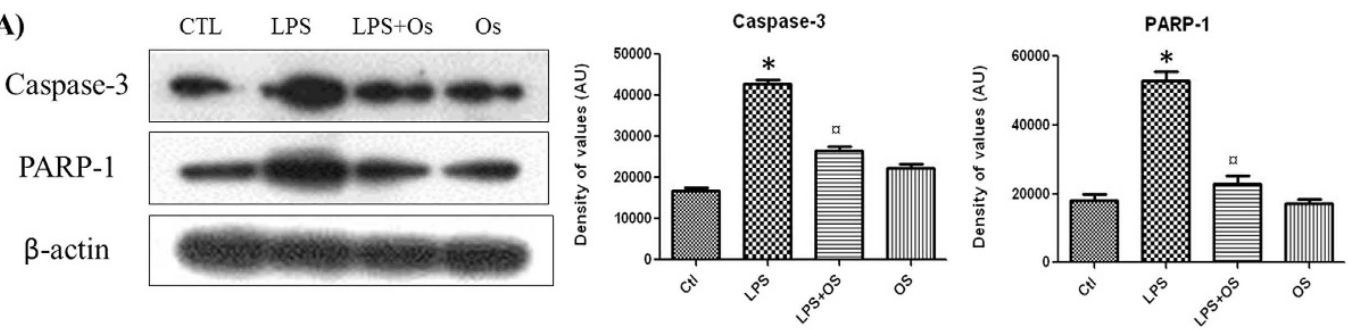

(B)
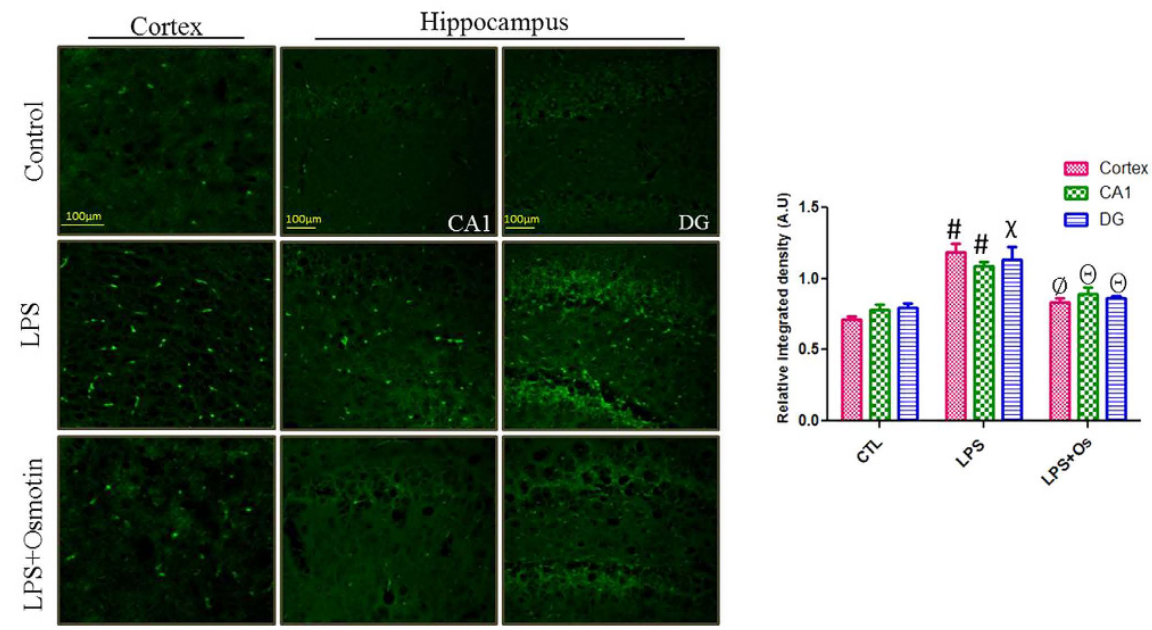

(C)

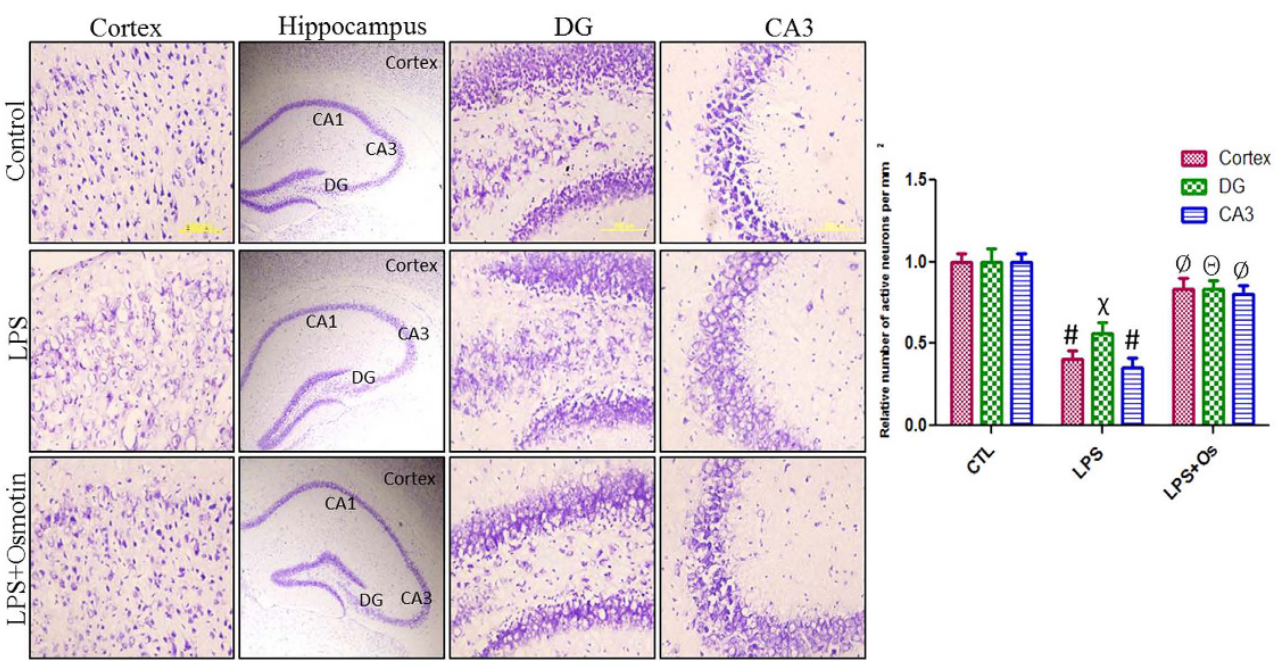

Figure 7. Osmotin attenuated LPS-induced apoptotic neurodegeneration in the hippocampus of adult mice (A) Shown are representative Western blots probed with caspase-3 and PARP-1 antibodies in the hippocampus of adult mice. The density values are expressed in arbitrary units as the mean $\pm \mathrm{SEM}$ for the indicated proteins ( $n=5$ animals per group). Shown are representative photomicrographs of (B) FJB staining (magnification 40x objective field, scale bar $=100 \mu \mathrm{m})$ and $(\mathbf{C})$ Nissl staining (magnification $20 \times$ objective field, scale bar $=200 \mu \mathrm{m}$ ) for dead and damaged neurons. Images are representative of staining obtained in sections prepared from at least 5 animals per group. Symbols for treatment groups and level of significance are mentioned in the data analysis section of the Materials and Methods.

mouse model. We hypothesized that osmotin would inhibit TLR4 downstream signaling via interaction with the TLR4-CD14 receptor complex and prevent neuroinflammation-induced neurodegeneration (Fig. S5).

Activation of TLR4 and CD14 receptors is the primary event in the induction of inflammatory processes. Pathogens, especially bacterial endotoxins, specifically recognize the TLR4 substrate, and it binds to TLR4 and $\mathrm{CD} 14$ receptors and triggers immune responses ${ }^{23,25}$. Activation of TLR4 signaling pathways induces NF $\kappa \mathrm{B}$ activation and triggers the release of inflammatory mediators, including COX-2, TNF- $\alpha$, IL-1, IL- 6 and iNOS ${ }^{2,36}$. LPS binds to TLR4 and CD14 receptors at the primary level and induces NFKB signaling pathway and subsequent cellular events ${ }^{37,38}$. TLR4 activation induces proinflammatory cytokines via the signaling of different adaptor proteins, such as TIRAP and MyD88, and activates NFKB and activator protein (AP)-1, which triggers 
the activation of other inflammatory genes ${ }^{36,39}$. Notably, recent studies demonstrated that adiponectin interfered with the TLR4-CD14 complex in cardiomyocytes via binding to AdipoR1 and prevented downstream signaling to inhibit inflammatory processes ${ }^{18}$. Adiponectin exhibits a promising role in inflammation-associated diseases, such as obesity, diabetes, cardiac injuries and neurological disorders ${ }^{40,41}$. Osmotin is a homologue of adiponectin, and we hypothesized that osmotin would prevent LPS-induced inflammatory processes in a similar manner as adiponectin. The established mechanisms of the beneficial effects of adiponectin or osmotin mostly describe AMPK and its related molecules. However, our study focused on the anti-inflammatory effect of osmotin via interaction with the TLR4-CD14 receptor complex. Previous studies demonstrated that the TLR4 complex contains several receptors, such as MD-2, CD14, TRIF, and MyD88, that are involved in signal processing ${ }^{39}$. Our results demonstrated the presence of AdipoR1 at the TLR4 site, and osmotin interacted with AdipoR1 to prevent the downstream inflammatory signals.

Chronic neuroinflammation is a pathological event that occurs during the progression of AD. Activation of neuronal microglia and reactive astrocytes during neuroinflammation are important components in $\mathrm{AD}$ progres$\operatorname{sion}^{42,43}$. The surrounding of $\beta$-amyloid deposits in $\mathrm{AD}$ brains may contain microlocalized acute phase reactant proteins, cytokines, and other inflammatory mediators ${ }^{12}$. Several studies confirmed impairments of cognitive function and increased levels of AD markers, such as A $\beta$, BACE-1, and p-Tau, following LPS administration in rodents ${ }^{4,16}$. Deng et al. ${ }^{15}$ recently demonstrated that LPS-induced chronic neuroinflammation developed an amyloidogenic axonal and dendritic pathology with up-regulated BACE-1 and A $\beta$ levels in adult rat brains ${ }^{15}$.

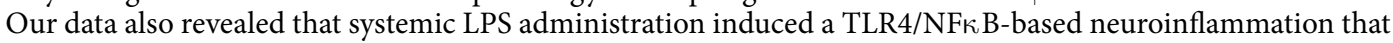
increased the levels of $\mathrm{A} \beta$, p-Tau, and BACE- 1 to the detriment of synaptic function. In contrast, several research studies reported an association of the TLR4/CD14 complex with innate immunity receptors on microglia that are involved in the clearance of $\mathrm{A} \beta$ deposits as a natural defense mechanism ${ }^{44,45}$. Recent research elucidated the mechanisms of the neuroprotective and anti-AD effects of adiponectin, which include protection against $\mathrm{A} \beta$-induced oxidative stress, the insulin-sensitizing action of adiponectin, the modulation of brain metabolism and its associated cognitive impairment, and inflammation-associated memory functions $\mathrm{s}^{46,47}$. The homolog of adiponectin, osmotin, also exhibited an anti-AD effect via a reduction in the protein expression levels of $A \beta$, APP, BACE-1, and p-Tau in adult mouse hippocampus (Fig. 6). We also demonstrated that osmotin regained synaptic functionality via elevation of the expression of pre- and post-synaptic proteins, such as SYP, SNAP-25, and PSD-95.

LPS-induced neuroinflammation activates the mitochondrial apoptotic pathway and enhances apoptotic neurodegeneration ${ }^{48}$. LPS-induced apoptosis was also widely investigated in other tissues, such as myocardial cells, endothelial cells, and hepatocytes ${ }^{49-51}$. In vitro and in vivo studies demonstrated that LPS induced neuronal cell death via a TLR4-dependent pathway ${ }^{30}$. TLR4 signaling leads to the activation of AP-1, and AP-1 promotes the JNK signaling cascade to induce neuroinflammation and neurodegeneration and/or interferes with the BCL-2 family of proteins to activate the mitochondrial apoptotic pathway ${ }^{52,53}$. Our previous reports demonstrated the neuroprotective and anti-apoptotic effects of osmotin in developmental models ${ }^{21,22}$. The present study investigated the potential anti-apoptotic behavior of osmotin in the hippocampus of adult mice. Our results demonstrated that osmotin inhibited LPS-induced DNA damage and activated the caspase cascade (Fig. 7). Overall, this study demonstrated the protective effect of osmotin against LPS-induced neuroinflammation and neurodegeneration in vivo and in vitro.

\section{Conclusion}

The findings of the current study support the use of osmotin as a novel therapeutic agent for the treatment of neuroinflammation, neurodegeneration, and associated diseases. This study provides a new strategy to target adiponectin receptors for the treatment of neurodegenerative disorders. Our results demonstrated that the binding of osmotin to AdipoR1 at the TLR4 site inhibited LPS-induced neuroinflammatory and neurodegenerative signals. However, further research is required to evaluate the mechanistic role of osmotin in various neurodegenerative disorders.

\section{Materials and Methods}

In vitro cell culture and drug treatment. BV2 microglial cells were kindly provided by Dr. I. W. Choi (Inje University, Busan, Korea). BV2 cells were maintained in a solution of DMEM, 10\% fetal bovine serum (FBS), and antibiotics (penicillin and streptomycin). BV2 cells were grown for 4 days and treated as follows: (1) Control: incubated in DMEM solution for $24 \mathrm{hr}$; (2) LPS-treatment: incubated in DMEM solution containing LPS $(1 \mu \mathrm{g} / \mathrm{ml})$ for $24 \mathrm{hr}$; (3) LPS + osmotin co-treatment: incubated in DMEM solution containing LPS $(1 \mu \mathrm{g} / \mathrm{ml})$ and osmotin $(0.4 \mu \mathrm{M})$ for $24 \mathrm{hr}$; (4) osmotin treatment: incubated in DMEM solution containing osmotin $(0.4 \mu \mathrm{M})$ for $24 \mathrm{hr}$; (5) TAK242 and LPS treatment: incubated in DMEM solution containing TAK242 (1 $\mu \mathrm{M})$ for $2 \mathrm{hr}$ and post-incubated with LPS $(1 \mu \mathrm{g} / \mathrm{ml})$ for $24 \mathrm{hrs}$; and (6) AdipoR1siRNA treatment: incubated in DMEM solution containing AdipoR1siRNA $(30 \mathrm{pM})$ for $48 \mathrm{hr}$ and post-incubated with LPS $(1 \mu \mathrm{g} / \mathrm{ml})$ and osmotin $(0.4 \mu \mathrm{M})$ for $24 \mathrm{hr}$. All groups were treated on day 4 , and cells were harvested on day 5 for use in the desired analyses.

ApoTox-Glo, triplex assay. The ApoTox-Glo triplex assay (Promega, Promega BioSciences, LLC, San Luis Obispo, CA, USA) was performed according to our previously defined procedure ${ }^{22}$. Briefly, the BV2 microglial cell lines were seeded in 96 -well plates $\left(1 \times 10^{5}\right.$ cells/well $)$ in $200 \mu \mathrm{l}$ of DMEM media. Cells received respective drug treatments, and viability/cytotoxicity reagents containing a GF-AFC substrate and bis-AAF-R110 substrate were added to all wells. Cells were agitated for $30 \mathrm{sec}$ and incubated for $1 \mathrm{hr}$ at $37^{\circ} \mathrm{C}$. Fluorescence spectra were measured at $400 / 505 \mathrm{~nm}$ for viability assays and $485 / 520 \mathrm{~nm}$ for cytotoxicity assays. Caspase $3 / 7$ activity was assessed by the addition of caspase-Glo $3 / 7$ reagent $(100 \mu \mathrm{l})$ to all wells, and luminescence was measured at 
$485 / 520 \mathrm{~nm}$ to determine caspase activation. The results were calculated as \% cell viability, \% cytotoxicity, and \% caspase-3/7 activity.

Western blot analysis. Western blot analyses for cell lines were performed to determine the protein expression levels of inflammatory proteins. Proteins samples from microglial cells were collected after centrifugation and separated on Novex 4-12\% Bis-Tris Plus gels (Life Technologies) under reducing conditions. Separated proteins were transferred to a polyvinylidene difluoride (PVDF) membrane. A broad range of protein markers (7-200 kDa; GangNam-STAIN, iNtRON Biotechnology) was run in parallel to detect the molecular weights of the proteins. Skimmed milk was used for membrane blocking to reduce non-specific binding. Immunoblottings were performed using respective primary antibodies and secondary antibodies. An anti-actin antibody served as the loading control. Immunocomplexes were visualized using Ez West Lumi western blotting detection reagent (Atto Corporation Tokyo). The X-ray films were scanned, and optical densities of the bands were measured using computer-based Sigma Gel software (Jandel Scientific, San Rafeal, Chicago, IL, USA).

Immunoprecipitation. Immunoprecipitation analyses were performed to determine interactions between TLR4, CD14, and AdipoR1 receptors. Briefly, microglial cells were collected using RIPA buffer, incubated at $4^{\circ} \mathrm{C}$ for $10 \mathrm{~min}$, disrupted and centrifuged at $10000 \times g$ to pellet cellular debris. Protein A/G plus-agarose beads $(20 \mu \mathrm{l})$ were added to the protein sample and incubated for $2 \mathrm{hrs}$. The beads were retrieved using centrifugation at $2500 \mathrm{rpm}$. The supernatants were removed, and $10 \mu \mathrm{l}$ of primary antibody was added (anti-TLR4, goat polyclonal, Santa Cruz Biotechnology). Samples were incubated at $4{ }^{\circ} \mathrm{C}$ overnight on a rotating device. Protein A/G agarose beads $(40 \mu \mathrm{l})$ were added and incubated for $4 \mathrm{hrs}$. Antibody-bound beads were collected using centrifugation (2500 rpm, $5 \mathrm{~min}$ ) and washed three times with PBS following repeated centrifugation. The final pellet was solubilized in $30 \mu \mathrm{l}$ of $2 \times$ electrophoresis sample buffer, and the solution was boiled for 3-4 min. Proteins were analyzed using Western blot analysis.

In vivo drug treatment. C657LB male mice $(\mathrm{n}=40$, Gyeongsang National University Animal Breeding Center, Jinju, South Korea) weighing 25-30g at the start of the treatment were housed in a temperature-controlled environment and maintained on a 12-hr light/dark cycle (lights on at 6:00 am) with food available ad libitum. The mice were randomly divided into 4 groups as described below.

1. Control group (i.p. saline for 1 week).

2. LPS group (i.p. $250 \mu \mathrm{g} / \mathrm{kg}$ for 1 week).

3. LPS + osmotin (i.p. $15 \mu \mathrm{g} / \mathrm{g}, 3$ times weekly).

4. Osmotin (i.p. $15 \mu \mathrm{g} / \mathrm{g}, 3$ times weekly).

Figure S4 describes the drug treatment schedule. Experimental procedures were performed in accordance with the rules established by the animal ethics committee (IACUC) of the Division of Applied Life Sciences, Department of Biology, Gyeongsang National University South Korea. All of the in vivo experimental techniques were approved (Approval ID: 125) by the animal ethics committee (IACUC) of the Division of Applied Life Sciences, Department of Biology at Gyeongsang National University, South Korea.

Behavioral studies (Morris water maze test). A spatial memory test was performed for behavioral studies of experimental mice. The water maze apparatus is a circular water tank (diameter: $100 \mathrm{~cm}$ and height: $40 \mathrm{~cm}$ ) filled with white ink-mixed water to a depth of $15.5 \mathrm{~cm}$ and maintained at $22-25^{\circ} \mathrm{C}$. The circular pool was divided into four equal quadrants, and a transparent escape platform (diameter: $10 \mathrm{~cm}$ and height $14.5 \mathrm{~cm}$ ) was placed at midpoint of one quadrant. The swimming behavior of the mice was monitored and analyzed using a video tracking system (SMART, Panlab Harward Apparatus, Bioscience Company, USA). The mice were trained for 4 consecutive days before treatment and rested for 3 days. The latency to escape was calculated for 6 consecutive days. The final escape latency and probe tests were performed on the $6^{\text {th }}$ day to evaluate consolidated spatial memory. The platform was removed during the probe trial, and each mouse was allowed to search for the platform in the pool for $60 \mathrm{sec}$. The time spent in the target quadrant was used to evaluate the degree of memory consolidation.

Western blot analysis. Mice were anesthetized after treatment and decapitated, and the brain samples were differentiated. Cortices and hippocampi were removed and frozen in dry ice. All brain tissues were homogenized in pro-prep extraction solution (iNtRON Biotechnology). Proteins were processed for immunoblotting as described above.

Tissue sample preparation. Mouse brain samples $(n=5$ per group) were collected after transcardial perfusion with normal saline solution $(0.9 \%)$ and $4 \%$ ice-cold paraformaldehyde. Brain tissues were fixed in paraformaldehyde for 3 days followed by immersion in a $20 \%$ sucrose phosphate buffer for 3 days. Brain tissues were frozen in O.C.T. compound (A.O. Co., USA), and 12-14- $\mu \mathrm{m}$ sections were created in coronal planes (Leica cryostat CM 3050, Germany). Sections were thaw-mounted on probe-on plus-charged slides (Fisher, USA) at room temperature and stored at $-70^{\circ} \mathrm{C}$.

Immunofluorescence assays. Tissue slides were dried overnight and washed twice with $0.01 \mathrm{M}$ PBS for $5 \mathrm{~min}$. Tissue samples were incubated with proteinase $\mathrm{K}$ for $5 \mathrm{~min}$, rinsed with PBS and blocked with normal

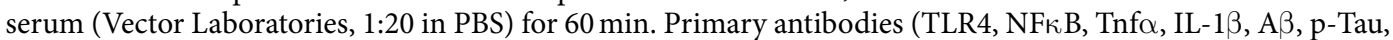
PSD95, and Snap-25) were diluted 1:100 in PBS containing 2\% serum and 0.1\% Triton X-100 and incubated at $4{ }^{\circ} \mathrm{C}$ overnight. FITC- or TRITC-labeled secondary antibodies (anti-rabbit, anti-goat, or anti-mouse) were diluted 
1:50 in PBS and applied at room temperature for $90 \mathrm{~min}$. Slides were washed twice with PBS for $5 \mathrm{~min}$. The primary and secondary antibodies for double immunofluorescence were applied the following day. Glass cover slips were mounted on glass slides using fluorescent mounting medium (Dako 53023). Images were captured using a confocal laser scanning microscope (FV 1000MPE, Olympus, Japan).

Fluoro Jade B staining. Fluoro-Jade B staining was performed according to the manufacturer protocol (cat\# AG310, Millipore, USA). The tissue slides were air-dried overnight and immersed in a solution of $1 \%$ sodium hydroxide and $80 \%$ ethanol for $5 \mathrm{~min}$. The slides were washed with $70 \%$ ethanol followed by distilled water for $2 \mathrm{~min}$ each. Then the slides were immersed in a solution of $0.06 \%$ potassium permanganate for $10 \mathrm{~min}$, rinsed with distilled water and transferred to a solution of $0.1 \%$ acetic acid and $0.01 \%$ fluoro-jade B for $20 \mathrm{~min}$. The slides were washed with distilled water and allowed to dry for $10 \mathrm{~min}$. Slices were cleared in xylene for $5 \mathrm{~min}$, glass cover slips were mounted on the slides and images were prepared with confocal laser scanning microscope (FV 1000, Olympus, Japan).

Nissl staining. Tissue samples from control and experimental groups were washed twice with $0.01 \mathrm{M}$ PBS for 5-10 min. Slides were stained with warmed a $0.5 \%$ cresyl violet solution for $10 \mathrm{~min}$, rinsed with distilled water, and dehydrated in a series of graded ethanol (70\%, 95\% and 100\%). Tissue slices were cleared in xylene for $5 \mathrm{~min}$, and glass cover slips were mounted using mounting medium. Images were captured using a fluorescent light microscope.

Data analysis and statistics. Western blot bands were scanned and analyzed using densitometry in the computer-based Sigma Gel System (SPSS Inc., Chicago, IL). Immunofluorescence results were evaluated using computer-based ImageJ software, and densities were calculated in arbitrary units. The data are presented as the mean \pm standard error of mean (SEM). Data were analyzed using ANOVA followed by Student's $t$-test. The symbol ' $*$ ' indicates a significant difference $(p<0.001)$, '”' indicates a significant difference $(p<0.01)$, and ' $\chi$ ' indicates a significant difference $(p<0.05)$ compared to their respective control groups. The symbol ' $x$ ' indicates a significant difference $(p<0.001)$, ' $\varnothing$ ' indicates a significant difference $(p<0.01)$, and ' $\Theta$ ' indicates a significant difference $(p<0.05)$ compared to LPS-treated groups. (Western blots and graph bars are represented as $\mathrm{CTL}=$ Control, LPS $=$ LPS treated group, LPS $+\mathrm{Os}=\mathrm{LPS}$ and osmotin treated group, and Os = Alone osmotin treated group).

\section{References}

1. Badshah, H. et al. Protective effect of lupeol against lipopolysaccharide-induced neuroinflammation via the p38/c-Jun N-terminal kinase pathway in the adult mouse brain J. Neuroimmune Pharmacol. 11, 48-60 (2016).

2. Parajuli, B. et al. GM-CSF increase LPS-induced production of proinflammatory mediators via upregulation of TLR4 and CD14 in murine microglia. J. Neuroinflammation 9, 268 (2012).

3. Font-Nieves, M. et al. Induction of COX-2 enzyme and down-regulation of COX-1 expression by lipopolysaccharide (LPS) control prostaglandin E2 production in astrocytes. J. Biol. Chem. 287, 6564-68 (2012).

4. Rosi, S. et al. Memantine protects against LPS-induced neuroinflammation, restores behaviourally-induced gene expression and spatial learning in the rat. Neuroscience 142, 1303-15 (2006).

5. Okun, E., Griffioen, K. J. \& Mattson, M. P. Toll-like receptor signaling in neural plasticity and disease. Trends Neurosci. 34, 269-81 (2011).

6. Garate, I. et al. Toll-like receptor inhibitor TAK242 decreases neuroinflammation in rat brain frontal cortex after stress. J. Neuroinflammation 11, 8 (2014).

7. Sambamurti, K. et al. Gene structure and organization of the human beta-secretase (BACE) promoter. FASEB J. 18, 1034-6 (2004).

8. Qin, L. et al. Systemic LPS causes chronic neuroinflammation and progressive neurodegeneration. Glia 55, 453-62 (2007).

9. Sastre, M. et al. Nonsteroidal anti-inflammatory drugs and peroxisome proliferatoractivated receptor-gamma agonists modulate immunostimulated processing of amyloid precursor protein through regulation of beta-secretase. J. Neurosci. 23, 9796-804 (2003).

10. McGeer, E. G. \& McGeer, P. L. Inflammatory processes in Alzheimer's disease. Prog. neuropsychopharmacol. Biol. Psychiatry 27, 741-9 (2003).

11. Pratico, D. \& Trojanowski, J. Q. Inflammatory hypotheses: novel mechanisms of Alzheimer's neurodegeneration and new therapeutic targets? Neurobiol. Aging 21, 441-3 (2000).

12. Akiyama, H. et al. Inflammation and Alzheimer's disease. Neurobiol. Aging 21, 383-421 (2000).

13. Griffin, W. S. et al. Glial-neuronal interactions in Alzheimer's disease: the potential role of a 'cytokine cycle' in disease progression. Brain Pathol. 8, 65-72 (1998).

14. Sambamurti, K. et al. Gene structure and organization of the human beta-secretase (BACE) promoter. FASEB J. 18, 1034-6 (2004).

15. Deng, X. et al. Lipopolysaccharide-induced neuroinflammation is associated with Alzheimer-like amyloidogenic axonal pathology and dendritic degeneration in rats. Adv. Alzheimer Dis. 3, 78-93 (2014).

16. Lee, Y. J. et al. Epigallocatechin-3-gallate prevents systemic inflammation-induced memory deficiency and amyloidogenesis via its anti-neuroinflammatory properties. J. Nutr. Biochem. 24, 296-310 (2013).

17. Teeling, J. L. et al. The effect of non-steroidal anti-inflammatory agents on behavioral changes and cytokine production following systemic inflammation: Implications for a role of COX-1. Brain Behav. Immun. 24, 409-19 (2010).

18. Jenke, A. et al. Adiponectin protects against Toll-like receptor 4-mediated cardiac neuroinflammation and injury. Cardiovas. Res. 99, 422-31 (2013).

19. Narasimhan, M. L. et al. Osmotin is a homolog of mammalian adiponectin and controls apoptosis in yeast through a homolog of a mammalian adiponectin receptor. Mol. Cell 17, 171-80 (2005).

20. Miele, M., Costantini, S. \& Colonna, G. Structural and functional similarities between osmotin from Nicotiana tabacum seeds and human adiponectin. PLos One 6, el6690 (2011).

21. Naseer, M. I. et al. Neuroprotective effect of osmotin against ethanol-induced apoptotic neurodegeneration in the developing brain. Cell Death Dis. 5, e1150 (2014).

22. Ali, T. et al. Osmotin attenuates amyloid beta-induced memory impairment, tau phosphorylation and neurodegeneration in the mouse hippocampus. Sci. Rep. 5, 11708 (2015).

23. Park, B. S. \& Lee, J. O. Recognition of lipopolysaccharide pattern by TLR4 complexes. Exp. Mol. Med. 45, e66 (2013).

24. Zanoni, I. et al. CD14 controls the LPS-induced endocytosis of Toll-like receptor 4. Cell 147, 868-80 (2011).

25. Gorina, R. et al. Astrocyte TLR4 activation induces a proinflammatory environment through the interplay between My88dependent NFאB signaling, MAPK, and Jak1/Stat1 pathways. Glia 59, 242-55 (2011). 
26. Delhase, M., Hayakawa, M., Chen, Y. \& Karin, M. Positive and negative regulation of IkappaB kinase activity through IKKbeta subunit phosphorylation. Science 284, 309-13 (1999).

27. Lawrence, T. The nuclear factor NF-kappa B pathway in inflammation. Cold Spring Harb. Perspect. Biol. 1, a001651 (2009).

28. Block, M. L., Zecca, L. \& Hong, J. S. Microglia-mediated neurotoxicity: uncovering the molecular mechanisms. Nat. Rev. Neurosci. 8, 57-69 (2007).

29. Brown, G. C. \& Neher, J. J. Inflammatory neurodegeneration and mechanisms of microglial killing of neurons. Mol. Neurobiol. 41, 242-7 (2010).

30. Lehnardt, S. et al. Activation of innate imunity in the CNS triggers neurodegeneration through a Toll-like receptor 4-dependent pathway. Proc. Natl. Acad. USA 100, 8514-9 (2003).

31. Marcello, E., Epis, R., Saraceno, C. \& Di Luca, M. Synaptic dysfunction in Alzheimer's disease. Adv. Exp. Med. Biol. 970, 573-601 (2012)

32. Xaus, J. et al. LPS induces apoptosis in macrophages mostly through the autocrine production of TNF-alpha. Blood 95, 3823-31 (2000)

33. Rupinder, S. K., Gurpreet, A. K. \& Manjeet, S. Cell suicide and caspases. Vascul. Pharmacol. 46, 383-39 (2007).

34. Koh, D. W., Dawson, T. M. \& Dawson, V. L. Poly(ADP-ribosyl)ation regulation of life and death in the nervous system. Cell. Mol. Life Sci. 62, 760-768 (2005)

35. Garden, G. A. \& Moller, T. Microglia biology in health and disease. J.Neuroimmune Pharmacol. 1, 127-137 (2006).

36. Kawai, T. \& Akira, S. The role of pattern-recognition receptors in innate immunity: update on Toll-like receptors. Nat. Immunol. 11, 373-384 (2010).

37. Lakhani, S. A. \& Boque, C. W. Toll-like receptor signaling in sepsis. Curr. Opin. Pediatr. 15, 278-82 (2003).

38. Chow, J. C. et al. Toll-like receptor-4 mediates lipopolysaccharide-induced signal transduction. J. Biol. Chem. 274, 10689-92 (1999).

39. Akira, S. \& Takeda, K. Toll-like receptor signalling. Nat. Rev. Immunol. 4, 499-511 (2004).

40. Tsao, T. S., Lodish, H. F. \& Fruebis, J. ACRP30, a new hormone controlling fat and glucose metabolism. Eur. J. Pharmacol. 440, 213-21 (2002).

41. Kadowaki, T. \& Yamauchi, T. Adiponectin and adiponectin receptors. Endocr. Rev. 26, 439-51 (2005).

42. Hwang, D. Y. et al. Alterations in behavior, amyloid beta-42, caspase-3, and Cox-2 in mutant PS2 transgenic mouse model of Alzheimer's disease. FASEB J. 16, 805-13 (2002).

43. Nguyen, M. D., Julien, J. P. \& Rivest, S. Innate immunity: The missing link in neuroprotection and neurodegeneration? Nat. Rev. Neurosci. 3, 216-227 (2002).

44. Tahara, K. et al. Role of toll-like receptor signalling in Abeta uptake and clearance. Brain 129, 3006-19 (2006).

45. Reed-Geaghan, E. G., Savage, J. C., Hise, A. G. \& Landreth, G. E. CD14 and toll-like receptors 2 and 4 are required for fibrillar A $\{$ beta $\}$-stimulated microglial activation. J. Neurosci. 29, 11982-92 (2009).

46. Chan, K. H. et al. Adiponectin is protective against oxidative stress induced cytotoxicity in amyloid-beta neurotoxicity. PLos One 7, e52354 (2012).

47. Oh, D. K., Ciaraldi, T. \& Henry, R. R. Adiponectin in health and disease. Diabetes Obes. Metab. 9, 282-289 (2007).

48. Hattori, Y. et al. Insights into sepsis therapeutic design based on the apoptotic death pathway. J. Pharmacol. Sci. 114, 354-65 (2010).

49. Cederbaum, A. I., Yang, L., Wang, X. \& Wu, D. CYP2E1 sensitizes the liver to LPS- and Tnf $\alpha$-induced toxicity via elevated oxidative and nitrosative stress and activation of ASK-1 and JNK mitogen-activated kinases. Int. J. Hepatol. 582790 (2012).

50. Munshi, N. et al. Lipopolysaccharide-induced apoptosis of endothelial cells and its inhibition by vascular endothelial growth factor. J. Immunol. 168, 5860-6 (2002).

51. Dong, M. et al. Chronic Akt activation attenuated lipopolysaccharide-induced cardiac dysfunction via Akt/GSK3 3 -dependent inhibition of apoptosis and ER stress. Biochem. Biophys. Acta 1832, 848-63 (2013).

52. Putcha, G. V. et al. JNK-mediated BIM phosphorylation potentiates BAX-dependent apoptosis. Neuron 19, 899-914 (2003).

53. Tournier, C. et al. Requirement of JNK for stress-induced activation of the cytochrome c-mediated death pathway. Science $\mathbf{2 8 8}$, 870-874 (2000).

\section{Acknowledgements}

This research was supported by the Pioneer Research Center Program through the National Research Foundation of Korea funded by the Ministry of Science, ICT \& Future Planning (2012-0009521).

\section{Author Contributions}

H.B. and M.O.K. designed the research study and wrote the manuscript. H.B. and T.A. performed western blots and confocal microscopy. M.O.K. is the corresponding author and holds all the responsibilities related to this manuscript.

\section{Additional Information}

Supplementary information accompanies this paper at http://www.nature.com/srep

Competing financial interests: The authors declare no competing financial interests.

How to cite this article: Badshah, H. et al. Osmotin attenuates LPS-induced neuroinflammation and memory impairments via the TLR4/NFKB signaling pathway. Sci. Rep. 6, 24493; doi: 10.1038/srep24493 (2016).

(c) (i) This work is licensed under a Creative Commons Attribution 4.0 International License. The images or other third party material in this article are included in the article's Creative Commons license, unless indicated otherwise in the credit line; if the material is not included under the Creative Commons license, users will need to obtain permission from the license holder to reproduce the material. To view a copy of this license, visit http://creativecommons.org/licenses/by/4.0/ 